\title{
Vigilancia de la resistencia a los antibacterianos en Argentina. Programa WHONET, 1995-1996
}

\author{
Alicia Rossi, ${ }^{1}$ Marta Tokumoto, ${ }^{2}$ Marcelo Galas, ${ }^{1}$ \\ Rolando Soloaga, ${ }^{2}$ Alejandra Corso ${ }^{1}$ y Red Nacional \\ de Laboratorios que participan en el Programa WHONET ${ }^{3}$
}

RESUMEN La Organización Mundial de la Salud ha puesto en marcha un programa para la vigilancia de la resistencia a los antibacterianos, denominado WHONET, que se desarrolló en Argentina mediante una red de 23 laboratorios de instituciones hospitalarias públicas y privadas sometidos a programas nacionales e internacionales de control de calidad. Entre enero de 1995 y diciembre de 1996 se determinó por el método de difusión en agar la sensibilidad a los antibacterianos de 16073 aislados clínicos consecutivos, siguiendo las recomendaciones del Comité Nacional de Estándares para Laboratorios Clínicos (National Committee for Clinical Laboratory Standards: NCCLS) de los Estados Unidos de América. Más de la mitad de los aislados urinarios de Escherichia coli fueron resistentes a la ampicilina, y más de $30 \%$ a la trimetoprima-sulfametoxazol. Cuando se comparó la sensibilidad de los aislados urinarios de pacientes ambulatorios y hospitalizados, se observó una marcada diferencia en los perfiles de actividad (porcentaje de microorganismos resistentes aislados en pacientes hospitalizados frente a pacientes ambulatorios) de la gentamicina ( $8 \%$ frente a $2 \%)$, la norfloxacina (6\% frente a 2\%) y las cefalosporinas de tercera generación (18\% frente a 10\%). Los aislados de Klebsiella pneumoniae recuperados de hemocultivos presentaron resistencia a las cefalosporinas de tercera generación y a la gentamicina en 71 y $60 \%$ de los casos, respectivamente. La proporción de Staphylococcus aureus resistentes a la oxacilina fue de 39\%. Cerca de la mitad de los aislados de Enterococcus spp. presentaron resistencia de alto nivel a los aminoglucósidos, pero no se detectó resistencia a los glicopéptidos. En nuestro medio, la ampicilina y la trimetoprima-sulfametoxazol no fueron apropiadas para el tratamiento de las diarreas; Shigella flexneri presentó mayor porcentaje de aislados resistentes a ambos fármacos (87 y $74 \%$, respectivamente) que Shigella sonnei (47 y $71 \%$, respectivamente). Se detectó resistencia a las cefalosporinas de tercera generación en $40 \%$ de los aislados de Salmonella spp. recuperados en hospitales pediátricos. En casos de meningitis bacteriana, las tasas de resistencia de Streptococcus pneumoniae a la penicilina (18\%) y de Haemophilus influenzae a la ampicilina (19\%) se situaron en el rango intermedio de las descritas en países americanos y europeos.

1 Servicio Antimicrobianos. Instituto Nacional de Enfermedades Infecciosas, Administración Nacional de Enfermedades Infecciosas (ANLIS) "Dr. C.G. Malbrán". Av. Vélez Sarsfield 563, Buenos Aires, Argentina.

2 Instituto de Cirugía Cardiovascular, Fundación Favaloro, Buenos Aires, Argentina.

3 Marta Altschuler y Blanca Gatti, Hospital "Sor María Ludovica" (La Plata); Nora Gómez, Hospital "Cosme Argerich" (Buenos Aires); Laura Galanternik y Miriam Vásquez, Hospital "Ricardo Gutiérrez" (Buenos Aires); Marta Tokumoto y Rolando
Soloaga, Fundación Favaloro (Buenos Aires); Adriana Di Bella, Hospital "Posadas" (Buenos Aires); Horacio Lopardo, Hospital de Pediatría "J. P. Garrahan" (Buenos Aires); Elsa Couto, Hospital "F. Muñiz" (Buenos Aires); Liliana Guelfand, Fleni (Buenos Aires); Francisco Salomone, Hospital "General San Martín" (Entre Ríos); José Tanaro, Hospital Centenario de Gualeguaychu (Entre Ríos); Adriana Pereyra y Noemí Moreno, Hospital "Gobernador Centeno" (La Pampa); Sandra Grenon, Hospital Provincial de Pediatría (Misiones); Clara Kremer, Hospital Provincial de Neuquén (Neu- quén); Juana Molina, Hospital Materno Infantil (Salta); María Cacace, Hospital "San Vicente de Paúl" (Salta); Hugo Castro, Hospital "Marcial Quiroga" (San Juan); Graciela Algorry, Hospital Regional "Río Gallegos" (Santa Cruz); Clara Mayoral, Hospital "Gutiérrez" (Santa Fe); Cristina Lura de Calafell, Hospital "J. B. Iturraspe" (Santa Fe); Emma Sutich, Facultad de Ciencias Bioquímicas (Santa $\mathrm{Fe})$; Norma Zalazar, Hospital Regional de Río Grande (Tierra del Fuego); Miguel Arévalo, Hospital Regional de Ushuaia (Tierra del Fuego); Humberto Musa, Clínica de Microbiología Médica (Tucumán). 
En la segunda mitad de este siglo, el uso de agentes antimicrobianos ha contribuido sustancialmente a reducir la morbilidad y mortalidad de las enfermedades infecciosas; sin embargo, la selección de bacterias con resistencia natural y la adquisición y diseminación de mecanismos de resistencia por parte de cepas inicialmente sensibles a estos fármacos han contribuido al aumento continuo de la prevalencia de microorganismos resistentes.

La Organización Mundial de la Salud (OMS) ha desarrollado un programa de vigilancia de la resistencia a los antimicrobianos, denominado WHONET $(1,2)$. Uno de sus principales objetivos es facilitar los estudios de prevalencia de la resistencia en cada unidad hospitalaria. La creación de redes de laboratorios permite monitorizar la diseminación de los distintos perfiles de resistencia. Utilizando un programa informático único para el tratamiento de los datos, es posible establecer tendencias regionales, detectar la emergencia de resistencias inusuales y aportar bases racionales para el uso de los antimicrobianos (3).

En Argentina, el Instituto Nacional de Enfermedades Infecciosas (INEI), Administración Nacional de Enfermedades Infecciosas (ANLIS) "Dr. C. G. Malbrán" ha convocado a 23 laboratorios representativos de casi todas las áreas geográficas del país para obtener datos a escala nacional y constituir un sistema de vigilancia de la resistencia a los antibacterianos.

Los laboratorios participantes están incorporados al Programa Nacional de Control de Calidad en Bacteriología y Micología, organizado por el INEI y el Ministerio de Salud y Acción Social de la Provincia de Buenos Aires. Por su parte, desde 1995 la OMS ha incorporado al programa WHONET el WHOQuality Control and Proficiency Testing Project, coordinado por un experto del Centro para el Control y la Prevención de Enfermedades de los EUA (CDC), Atlanta, Georgia. Las cepas remitidas por dicho organismo internacional se derivan a los laboratorios participantes en la red nacional argentina.

El programa informático se distribuye libremente a todos los centros públicos o privados que estén interesados y la incorporación de estos a la red nacional se ajusta al diseño del muestreo y a la capacidad operativa del grupo coordinador.

En este estudio se presentan los perfiles de actividad de los antimicrobianos de mayor interés clínico frente a microorganismos causantes de infecciones urinarias, bacteriemia, diarrea y meningitis, observados durante los años 1995 y 1996 en Argentina.

\section{MATERIALES Y MÉTODOS}

Las características y distribución geográfica de los centros pertenecientes a la red fueron las siguientes (entre paréntesis se indica el número de instituciones participantes por cada provincia): 13 hospitales generales de Buenos Aires (3), Entre Ríos (2), La Pampa (1), Neuquén (1), Salta (1), San Juan (1), Santa Cruz (1), Santa Fe (1) y Tierra del Fuego (2); 7 hospitales pediátricos de Buenos Aires (2), La Plata (1), Misiones (1), Salta (1) y Santa Fe (2) y 3 laboratorios privados de Buenos Aires (2) y Tucumán (1). Todos los centros participantes han obtenido resultados satisfactorios en los programas nacionales e internacionales de control de calidad.

\section{Determinación de la sensibilidad a los antimicrobianos}

Se analizaron los perfiles de sensibilidad de 16073 aislados clínicos consecutivos provenientes de muestras de orina (11909), sangre (1948), heces (2 067) y líquido cefalorraquídeo (149).

Las especies bacterianas estudiadas fueron: Escherichia coli, Klebsiella pneumoniae, Proteus mirabilis, Enterobacter cloacae, Morganella morganii, Citrobacter freundii y Pseudomonas aeruginosa en las muestras de orina; E. coli, K. pneumoniae, Enterobacter cloacae, Salmonella spp., P. aeruginosa, Acinetobacter spp., Staphylococcus aureus, Streptococcus pneumoniae y Enterococcus spp. en las muestras de sangre; Shigella sonnei, Shigella flexneri y Salmonella spp. en las muestras de heces, y S. pneumoniae y
Haemophilus influenzae en las muestras de líquido cefalorraquídeo.

Se registraron los diámetros de las zonas de inhibición obtenidos con el método de difusión con discos, de acuerdo con las recomendaciones del Comité Nacional de Estándares para Laboratorios Clínicos (National Committee for Clinical Laboratory Standards: NCCLS). Para evaluar la sensibilidad de H. influenzae se utilizó el medio HTM (Haemophilus test medium). En todos los demás casos se utilizó agar de MuellerHinton, al cual se añadió 5\% de sangre de carnero en el caso de S. pneumoniae.

Las placas se inocularon con una suspensión bacteriana equivalente al 0,5 de la escala de McFarland y, después de colocar los monodiscos correspondientes, fueron incubadas a $35{ }^{\circ} \mathrm{C}$ durante 18 a 24 horas. Los discos con antibióticos utilizados en las pruebas de sensibilidad fueron de origen nacional (Laboratorios Britania y Servicio Antimicrobiano del INEI) o importados (Difco u Oxoid). Los resultados fueron interpretados con los criterios propuestos por el NCCLS en enero de 1997 (4).

Para evaluar la actividad de la cefotaxima y ceftazidima frente a aislados de E. coli y K. pneumoniae se aplicaron las recomendaciones del NCCLS de 1995 (5) y 1997 (4) para las pruebas de difusión. Las primeras consideran sensibles los aislados con halos de inhibición $\geq 18 \mathrm{~mm}$ con ceftazidima $\mathrm{y} \geq 23$ mm con cefotaxima (5), y las segundas consideran probables productores de $\beta$-lactamasas de espectro extendido (BLEE) los aislados con halos de inhibición $\leq 22$ con ceftazidima $\mathrm{y} \leq 27 \mathrm{~mm}$ con cefotaxima (4).

Frente a Enterococcus spp., la resistencia de alto nivel a los aminoglucósidos se determinó con discos de alta carga: $120 \mu \mathrm{g}$ de gentamicina y $300 \mu \mathrm{g}$ de estreptomicina.

Se evaluaron aquellos fármacos que representan una alternativa terapéutica y, para evitar el muestreo sesgado, no se incorporaron los resultados de determinaciones complementarias con antimicrobianos de amplio espectro realizadas únicamente en un grupo de aislados (por ejemplo, aquellos en los que se espera o se detecta multirresis- 
tencia). Para evaluar la sensibilidad de las enterobacterias a las cefalosporinas de tercera generación se utilizaron siempre cefotaxima y ceftazidima; la resistencia a alguna de las dos se interpretó como resistencia a ambas.

En el análisis estadístico se utilizó la prueba de $j i$ al cuadrado $\left(\chi^{2}\right)$ con corrección de Yates.

\section{Población}

Para evitar el análisis de microorganismos con idéntico antibiotipo provenientes de distintas muestras de un mismo individuo, solo se tuvo en cuenta un aislado por paciente; cuando se observó un cambio en el perfil de resistencia que implicara una modificación en la interpretación de los resultados, se consideró un aislado por cada perfil de resistencia observado. En el análisis de las infecciones urinarias los aislados se agruparon según procedieran de pacientes ambulatorios $\mathrm{u}$ hospitalizados.

\section{RESULTADOS}

El cuadro 1 muestra la actividad de varios antimicrobianos frente a microorganismos causantes de infecciones urinarias; E. coli fue responsable de $59,4 \%$ de las detectadas en pacientes hospitalizados y de 70,9\% de las detectadas en pacientes ambulatorios (datos no mostrados). La actividad de la ampicilina, ampicilina-sulbactam y trimetoprima-sulfametoxazol no fue significativamente diferente entre los aislados provenientes de uno y otro grupo de pacientes. En cambio, la proporción de aislados resistentes a la gentamicina, las fluoroquinolonas y las cefalosporinas de primera y tercera generación fue significativamente mayor $(P<0,001)$ en los pacientes hospitalizados. Esta diferencia también se observó con otros uropatógenos como K. pneu-

CUADRO 1. Porcentajes de resistencia a los antimicrobianos en microorganismos causantes de infecciones urinarias en pacientes hospitalizados y ambulatorios. Argentina, 1995-1996

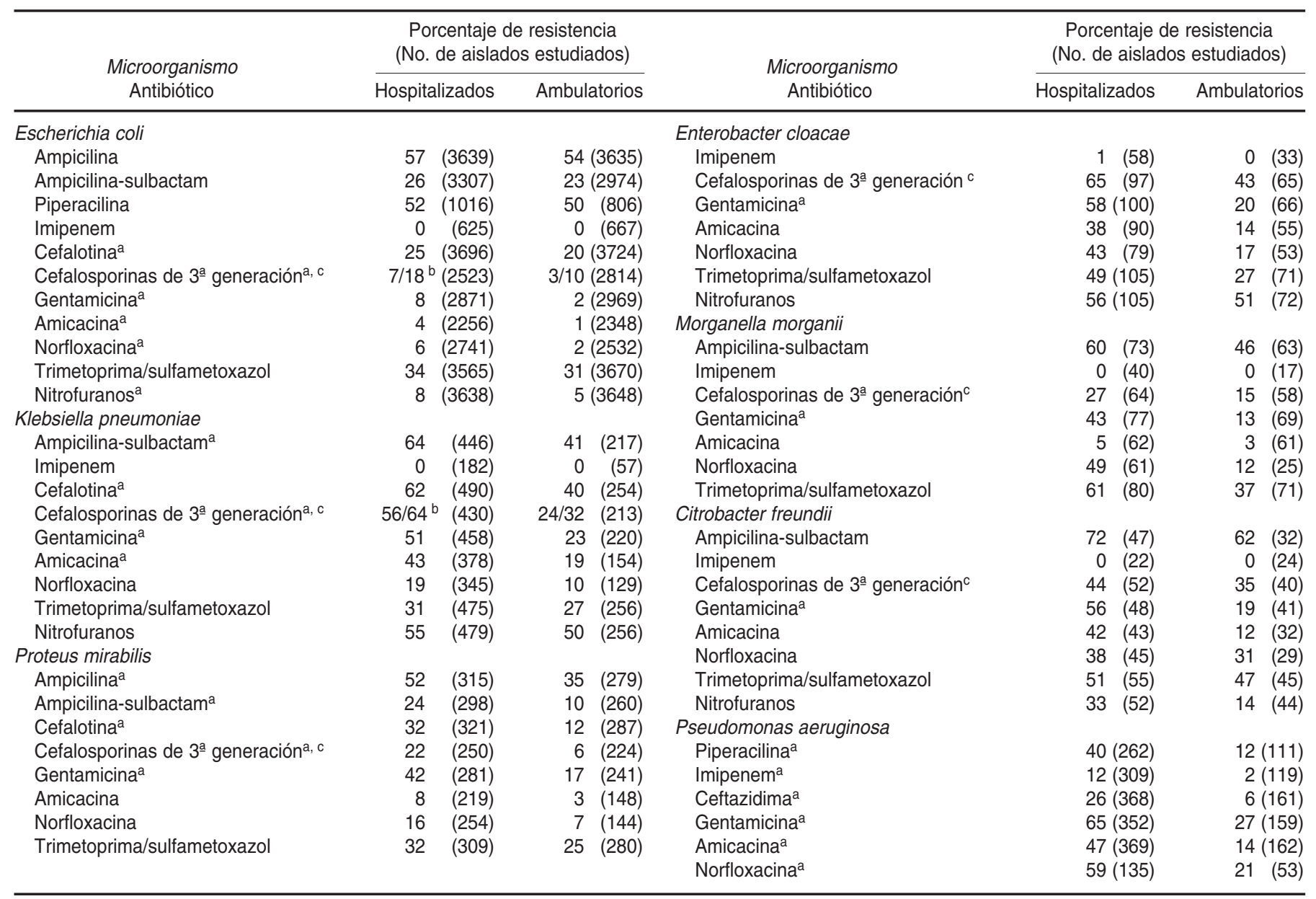

a Antibióticos que presentaron diferencias significativas entre los aislados procedentes de pacientes ambulatorios y hospitalizados $(P<0,001)$.

${ }^{b}$ Resultados obtenidos con los criterios de interpretación propuestos por el NCCLS en 1995/ resultados obtenidos con los criterios propuestos por el NCCLS en 1997 (ver Materiales y Métodos).

c Se ensayaron la cefotaxima y la ceftazidima. 
CUADRO 2. Porcentajes de resistencia a los antimicrobianos en bacilos gram-negativos recuperados por hemocultivo. Argentina, 1995-1996

\begin{tabular}{|c|c|c|c|c|c|c|c|c|c|c|}
\hline \multirow{2}{*}{$\begin{array}{l}\text { Microorganismos } \\
\qquad(n)\end{array}$} & \multicolumn{10}{|c|}{ Antimicrobianos $^{a}$} \\
\hline & AMP & AMS & PIP & IMI & CTN & CTG & GEN & AMI & CIP & TSX \\
\hline Escherichia coli (272) & 54 & 30 & 39 & 0 & 28 & $14^{\mathrm{b}}$ & 11 & 6 & 6 & 34 \\
\hline Klebsiella pneumoniae (251) & - & 69 & 77 & 0 & 72 & $71^{\mathrm{b}}$ & 60 & 49 & 11 & 34 \\
\hline Enterobacter cloacae (69) & - & - & 36 & 1 & - & $35^{b}$ & 27 & 18 & 11 & 28 \\
\hline Salmonella spp. (49) & 29 & 26 & 25 & 0 & - & $20^{\mathrm{b}}$ & 15 & 14 & 0 & 21 \\
\hline Pseudomonas aeruginosa (132) & - & - & 23 & 17 & - & $19^{c}$ & 36 & 22 & 19 & - \\
\hline Acinetobacter spp (100) & - & 32 & 74 & 1 & - & $54^{\mathrm{c}}$ & 51 & 53 & 64 & 55 \\
\hline
\end{tabular}

aMP: ampicilina; AMS: ampicilina-sulbactam; PIP: piperacilina; IMI: imipenem; CTN: cefalotina; CTG: cefalosporinas de tercera generación; GEN: gentamicina; AMI: amicacina; CIP: ciprofloxacina; TSX: trimetoprima-sulfametoxazol.

b Se ensayaron la cefotaxima y la ceftazidima.

${ }^{\mathrm{C}}$ Se ensayó la ceftazidima.

CUADRO 3. Porcentajes de resistencia a los antimicrobianos en cocos gram-positivos recuperados por hemocultivo. Argentina, 1995-1996

\begin{tabular}{|c|c|c|c|c|c|c|c|c|c|c|}
\hline \multirow{2}{*}{$\begin{array}{l}\text { Microorganismos } \\
(n)\end{array}$} & \multicolumn{10}{|c|}{ Antimicrobianos $^{a}$} \\
\hline & PEN & AMP & AMS & VAN & ERI & RIF & GEN & STR & TSX & CIP \\
\hline \multicolumn{11}{|l|}{ Staphylococcus aureus } \\
\hline MET S (431) & 92 & - & 2 & 0 & 15 & 6 & 9 & - & 1 & 2 \\
\hline Staphylococcus aureus & & & & & & & & & & \\
\hline MET R (275) & - & - & - & 0 & 76 & 68 & 94 & - & 56 & 65 \\
\hline $\begin{array}{l}\text { Streptococcus pneumoniae } \\
(191)\end{array}$ & $23^{b}$ & - & - & 0 & 6 & 6 & - & - & $\mathrm{Nd}^{\mathrm{c}}$ & $\mathrm{Nd}$ \\
\hline $\begin{array}{l}\text { Enterococcus spp. } \\
(178)\end{array}$ & - & 8 & 8 & 0 & - & - & $46^{d}$ & $51^{d}$ & - & - \\
\hline
\end{tabular}

a PEN: penicilina; AMP: ampicilina; AMS: ampicilina-sulbactam; VAN: vancomicina; ERI: eritromicina; RIF: rifampicina; GEN: gentamicina; STR: estreptomicina; TSX: trimetoprima-sulfametoxazol; CIP: ciprofloxacina; MET S: sensible a la meticilina; MET R: resistente a la meticilina.

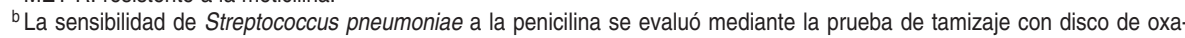
cilina $(1 \mu \mathrm{g})$.

Nd: No determinado.

${ }^{\mathrm{d}}$ Resistencia de alto nivel, determinada con discos con $120 \mu \mathrm{g}$ de gentamicina y $300 \mu \mathrm{g}$ de estreptomicina.

CUADRO 4. Porcentajes de resistencia a los antimicrobianos en microorganismos recuperados por coprocultivo. Argentina, 1995-1996

\begin{tabular}{lllllr}
\hline \multirow{2}{*}{$\begin{array}{c}\text { Microorganismos } \\
(n)\end{array}$} & \multicolumn{5}{c}{ Antimicrobianos $^{\mathrm{a}}$} \\
\cline { 2 - 6 } & AMP & FQ & CLO & TSX & CTG $^{\text {b }}$ \\
\hline Shigella flexneri (1183) & 87 & 0 & 59 & 74 & 2 \\
Shigella sonnei (382) & 47 & 0 & 5 & 71 & 1 \\
Salmonella spp.c (366) & 46 & 0 & 2 & 33 & 40 \\
Salmonella spp. $^{\mathrm{d}}(86)$ & 14 & 2 & 4 & 12 & 10 \\
\hline
\end{tabular}

a AMP: ampicilina; FQ: fluoroquinolonas (ciprofloxacina, norfloxacina); CLO: cloranfenicol; TSX: trimetoprima-sulfametoxazol; CTG: cefalosporinas de $3^{a}$ generación.

b Se ensayaron la cefotaxima y la ceftazidima.

${ }^{c}$ Aislados procedentes de hospitales pediátricos, de los cuales $58 \%$ corresponden a pacientes hospitalizados.

dAislados procedentes de hospitales generales, de los cuales $76 \%$ corresponden a pacientes hospitalizados. moniae y $P r$. mirabilis y fue aun más marcada con $P$. aeruginosa. Los aislados de Enterobacter cloacae, Morganella morganii y Citrobacter freundii solo presentaron diferencias significativas entre ambos grupos de pacientes en relación con la actividad de la gentamicina.

En los cuadros 2 y 3 se muestra la actividad de los antimicrobianos frente a aislados obtenidos por hemocultivo. Una proporción considerable de pacientes con bacteriemia o septicemia presentaron escasas alternativas terapéuticas. E. coli y K. pneumoniae presentaron resistencia muy elevada a las cefalosporinas de tercera generación y a los aminoglucósidos. También fue preocupante que $17 \%$ de los aislados de $P$. aeruginosa fueran resistentes al imipenem (cuadro 2).

La proporción de aislados de Staph. aureus resistentes a la oxacilina fue de $39 \%$, pero varió considerablemente según la institución analizada y, como está descrito a nivel internacional, la resistencia a la oxacilina se asoció fuertemente con la resistencia a la tetraciclina, los macrólidos, la rifampicina y los aminoglucósidos (cuadro 3). En Argentina, 94 y 76\% de los aislados resistentes a la meticilina también fueron resistentes a la gentamicina y eritromicina, respectivamente. La resistencia a la trimetoprima-sulfametoxazol y a las fluoroquinolonas estuvo presente casi exclusivamente en los aislados resistentes a la meticilina. Aproximadamente la mitad de los aislados de Enterococcus spp. causantes de bacteriemia o septicemia ( $n=178$ ) presentaron resistencia de alto nivel a los aminoglucósidos, pero no mostraron halos de inhibición $<17 \mathrm{~mm}$ con vancomicina.

La resistencia en bacterias causantes de diarrea se muestra en el cuadro 4. Shigella flexneri presentó tasas de resistencia a la ampicilina muy superiores a las de Shigella sonnei y cerca de $60 \%$ de los aislados fueron, además, resistentes al cloranfenicol. Más de $70 \%$ de los aislados de ambas especies fueron resistentes a la trimetoprima-sulfametoxazol. Los aislados de salmonelas recuperados en hospitales generales fueron en su mayoría sensibles a los antimicrobianos ensayados, mientras que en los hospitales pediátricos la resistencia 
FIGURA 1. Distribución de las zonas de inhibición obtenidas con ceftazidima y cefotaxima frente a Proteus mirabilis $(\mathrm{n}=698)$. Argentina, 1995-1996

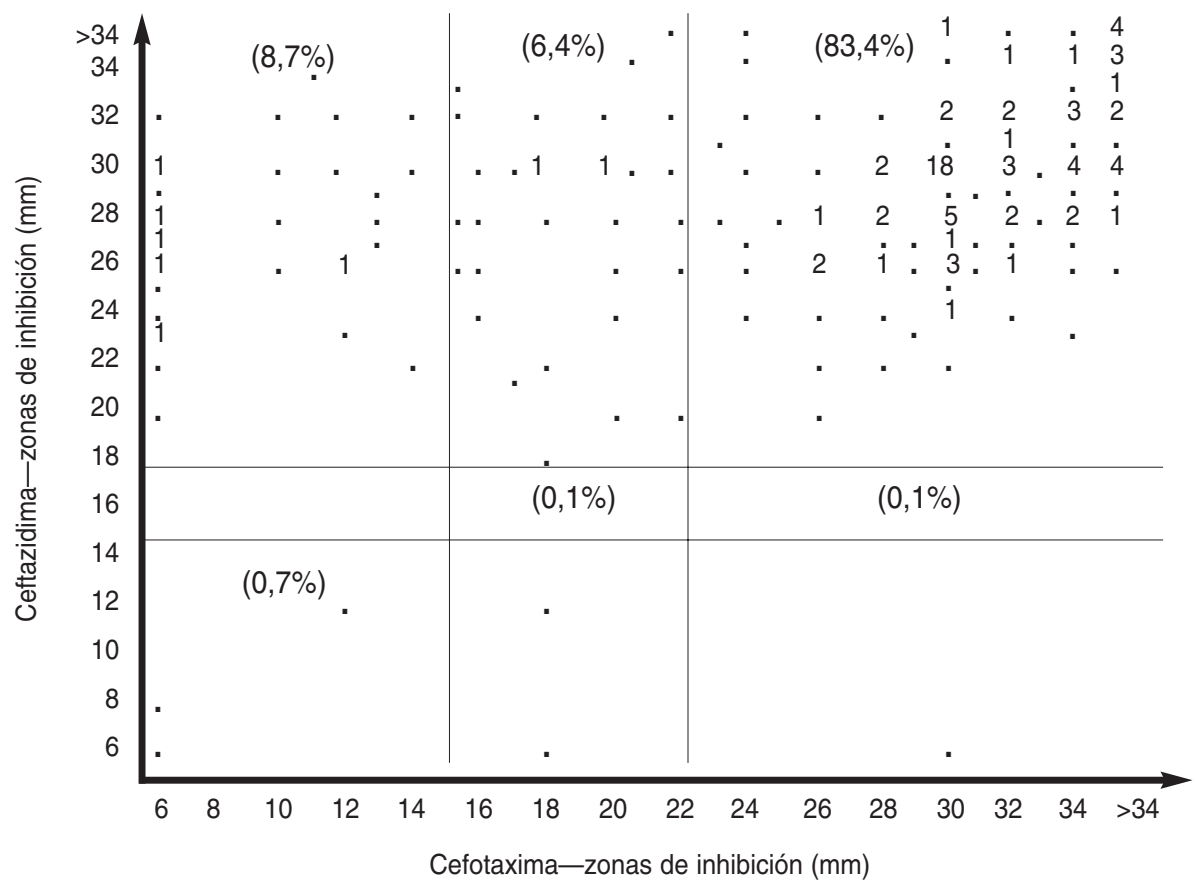

Puntos de corte: Cefotaxima $=15-22 \mathrm{~mm}$; Ceftazidima $=15-17 \mathrm{~mm}$. Los números indican porcentajes y los puntos, porcentajes $<1$. Se indican entre paréntesis los porcentajes totales de cada cuadrante.

FIGURA 2. Distribución de las zonas de inhibición obtenidas con ceftazidima y cefotaxima frente a Klebsiella pneumoniae $(\mathrm{n}=1160)$. Argentina, 1995-1996

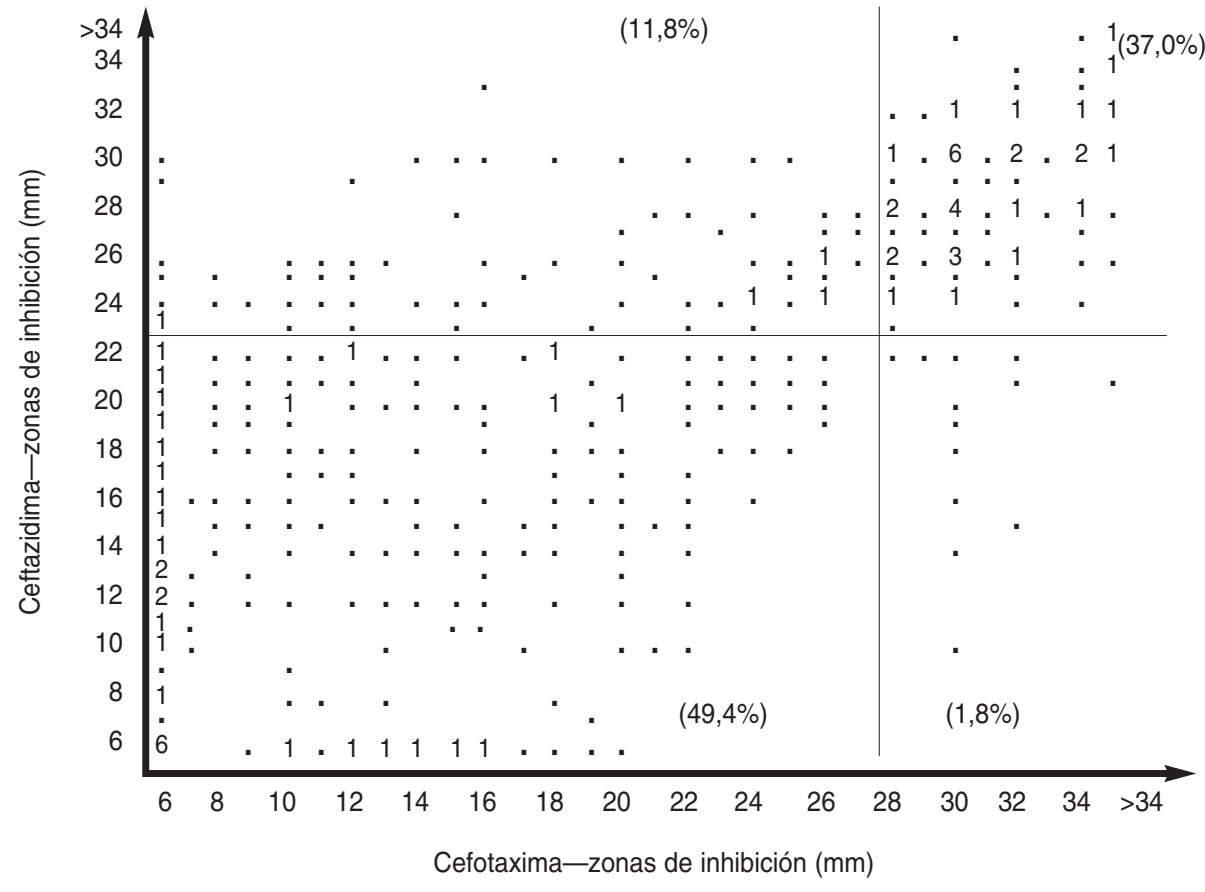

Puntos de corte: Cefotaxima $=\mathrm{S} \geq 28 \mathrm{~mm}$; Ceftazidima $=\mathrm{S} \geq 23 \mathrm{~mm}$. Los números indican porcentajes y los puntos, porcentajes $<1$. Se indican entre paréntesis los porcentajes totales de cada cuadrante. a las cefalosporinas de tercera generación afectó a $40 \%$ de los aislados.

Con respecto a la detección de BLEE en enterobacterias, las figuras 1 y 2 muestran una correlación entre los diámetros de inhibición observados con discos de cefotaxima y de ceftazidima frente a todos los aislados de K. pneumoniae y Pr. mirabilis; $11,8 \%$ de los aislados de K. pneumoniae y 15,1\% de los de Pr. mirabilis se manifestaron sensibles a la ceftazidima pero resistentes a la cefotaxima. En todos los cuadros, únicamente se consideraron sensibles a las cefalosporinas de tercera generación los microorganismos ubicados en el cuadrante superior derecho de figuras como la 1 y la 2 (aislados sensibles a ambos compuestos).

Como se muestra en el cuadro 5 , $18 \%$ de los aislados de S. pneumoniae causantes de meningitis fueron resistentes a la penicilina y $19 \%$ de los aislados de $H$. influenzae fueron resistentes a la ampicilina, por producción de $\beta$-lactamasas; la resistencia de este microorganismo al cloranfenicol (11\%) estuvo siempre asociada con la resistencia enzimática a la penicilina.

\section{DISCUSIÓN}

Como se muestra en el cuadro 1, las modificaciones en los puntos de corte para la cefotaxima y la ceftazidima (criterios del NCCLS de 1995 y de 1997) produjeron cambios importantes en el perfil de actividad de estos fármacos, particularmente frente a E. coli. En esta especie, los mecanismos más frecuentes de resistencia a las cefalosporinas de tercera generación están relacionados con la presencia de BLEE y, en menor proporción, con la hiperproducción de su $\beta$-lactamasa cromosómica (tipo AMP-C). El primer mecanismo está frecuentemente asociado a la resistencia a la gentamicina y el segundo puede sospecharse en los aislados con sensibilidad reducida a la cefoxitina. Siguiendo estos criterios, pudo estimarse que, frente a E. coli, los cambios en los puntos de corte incorporaron como posibles productores de BLEE a cepas que en su mayoría serían hiperproductoras de $\beta$-lactamasas cromosómicas del tipo 
CUADRO 5. Porcentajes de resistencia a los antimicrobianos en microorganismos causantes de meningitis bacteriana. Argentina, 1995-1996

\begin{tabular}{lcccccr}
\hline \multirow{2}{*}{$\begin{array}{c}\text { Microorganismos } \\
(n)\end{array}$} & \multicolumn{7}{c}{ Antimicrobianos $^{\mathrm{a}}$} \\
\cline { 2 - 7 } & PEN $^{\mathrm{b}}$ & AMP & AMS & CTX & RIF & CLO \\
\hline $\begin{array}{l}\text { Streptococcus pneumoniae (91) } \\
\text { Haemophilus influenzae (58) }\end{array}$ & 18 & - & - & - & 4 & 7 \\
\hline
\end{tabular}

a PEN: penicilina; AMP: ampicilina; AMS: ampicilina-sulbactam; CTX: cefotaxima; RIF: rifampicina; CLO: cloranfenicol.

b La sensibilidad de Streptococcus pneumoniae a la penicilina se evaluó mediante la prueba de tamizaje con disco de oxacilina $(1 \mu \mathrm{g})$

${ }^{c} \mathrm{Nd}$ : No determinado.

AMP-C. Estudios iniciales de caracterización molecular de estos aislados confirman esta estimación.

Los aislados de E. coli y Pr. mirabilis resistentes a la ampicilina y las demás enterobacterias resistentes a la ticarcilina se consideran como probables productores de $\beta$-lactamasas del tipo TEM-1, TEM-2 o SHV-1. Estas enzimas son capaces de hidrolizar, además, a las acilureidopenicilinas y a las cefalosporinas de primera generación, aunque esta actividad no siempre se refleja en el antibiograma. Algunos autores (6) recomiendan considerar a estos microorganismos como resistentes a la mezlocilina, piperacilina y cefalotina, independientemente de los resultados de las pruebas de sensibilidad a estos antibióticos, excepto en las infecciones de las vías urinarias bajas (7), en las que pueden ser eficaces. De esta manera, las actividades de la cefalotina y piperacilina frente a $E$. coli mostradas en el cuadro 2 serían aun menores y comparables a la de la ampicilina.

Aunque no se detectaron enterococos resistentes a la vancomicina, debe prestarse especial atención a la aparición de microorganismos con resistencia adquirida a los glicopéptidos, particularmente después de la reciente descripción en nuestro medio de los primeros casos de infecciones causadas por enterococos resistentes a la vancomicina. En Buenos Aires se han detectado dos aislados de Enterococcus faecalis resistentes a la vancomicina, el primero en enero de 1996, portador del mecanismo VAN B, y el segundo, aislado 14 meses después, con mecanismo aún no identificado. ${ }^{4}$ También en Buenos Aires, en enero de 1997 se identificaron tres aislados de Enterococcus faecium resistente a la vancomicina $y$ teicoplanina con idéntico perfil de restricción por electroforesis de campo pulsado (PFGE). ${ }^{5}$ En el mismo período se detectaron en Mendoza (a más de 1000 km de Buenos Aires), cinco aislados de Enterococcus faecium resistentes que, analizados por PFGE, mostraron estar estrechamente relacionados. ${ }^{6}$

Debido a la alta proporción de aislados resistentes, en nuestro medio la ampicilina y la trimetoprima-sulfametoxazol no son fármacos útiles para el tratamiento empírico inicial de las diarreas por Shigella spp. Las fluoroquinolonas presentaron gran actividad frente a estos microorganismos, pero aún no están recomendadas en pediatría. Otros fármacos menos tóxicos, como las cefalosporinas de primera generación o los aminoglucósidos, no son útiles clínicamente, a pesar de su aparente actividad in vitro.

\footnotetext{
4 Casellas JM, Tomé G, Lopez Furst M, Rolón MJ Alug J, Marcopiddo M, et al. Aislamiento de cepas de Enterococcus faecalis resistentes a vancomicina en Argentina. En: I Congreso Internacional de Infectología y Microbiología Clínica. Buenos Aires, 1997. Abstract H369.

5 Targa L, Carbone E, Gallego V, Podestá O, Marín M, Arduino R. Brote epidémico por Enterococcus faecium resistentes a vancomicina (ERV) en un hospital de Buenos Aires. En: I Congreso Internacional de Infectología y Microbiología Clínica. Buenos Aires, 1997. Abstract H330.

6 Podestá OS, Mera JR, Marín ME, Galdón F, Correa $\mathrm{AP}$, Stamboulian D et al. Brote de enterococos resistentes a la vancomicina (ERV) en un servicio de clínica médica en un hospital de Mendoza. En: I Congreso Internacional de Infectología y Microbiología Clínica. Buenos Aires, 1997. Abstract H332.
}

Desde hace casi una década, se registran en nuestros hospitales pediátricos brotes de infecciones intrahospitalarias por salmonelas multirresistentes. Un estudio de aislados de distintas serovariedades y representativos de varias áreas geográficas del país mostró en todos los casos la producción de una BLEE (CTX-M-2), y al menos dos enzimas inactivadoras de aminoglucósidos (acetilasa 3, tipo II y acetilasa 6, tipo I). Los genes correspondientes se localizaron en un plásmido conjugativo de alto peso molecular $(8)^{7}$. Estos genes de resistencia han tenido una difusión tan importante en nuestro país que hasta han sido detectados en aislados de $\mathrm{Vi}$ brio cholerae durante el segundo y sexto brote epidémico de cólera en Argentina (9). ${ }^{8}$ Como se muestra en el cuadro 4, el perfil de resistencia conferido por este tipo de enzimas está asociado a hospitales pediátricos.

A diferencia de lo descrito en recomendaciones internacionales (4), en nuestro país la cefotaxima fue superior a la ceftazidima para predecir la posible presencia de BLEE. Esta diferencia puede deberse a que, mientras en otras regiones la mayor parte de los genes de resistencia que codifican BLEE han emergido por mutación de genes conocidos $\left(b l a_{\text {tem }}\right.$ y bla $\left.a_{\text {shv }}\right)$, estudios iniciales realizados por el INEI mostraron que en nuestro medio predominan enzimas como CTX-M-2 y, en menor medida, derivadas de PER-2 y SHV (10). ${ }^{5}$ Estas enzimas, transferibles por conjugación, no han sido detectadas en otras áreas geográficas y solo se han detectado miembros de estas familias en Turquía y Alemania $(11,12)$. En enterobacterias productoras de estas enzimas, la resistencia a una cefalosporina de tercera generación indica resistencia al resto de los compuestos

\footnotetext{
Melano R, Petroni A, Galas M, Corso A, Viale D, Rossi A. Diseminación de la resistencia a cefalosporinas de tercera generación, en aislamientos de Salmonella spp. En: XIII Congreso Latinoamericano de Microbiología. Caracas, Venezuela. 1996. Abstract 224.

8 Galas M, Petroni A, Melano R, Corso A, Couto E, Blazquez J, et al. Caracterización de PER-2, una $\beta$-lactamasa de espectro extendido presente en enterobacterias y Vibrio cholerae. En: XIII Congreso Latinoamericano de Microbiología. Caracas, Venezuela. 1996. Abstract 225.
} 
de ese grupo, a las cefalosporinas de cuarta generación y al aztreonam.

Los porcentajes de resistencia a las penicilinas en microorganismos causantes de meningitis se situaron en el rango intermedio de los descritos en países americanos y europeos durante el mismo período.

En resumen, mientras que los niveles de resistencia de los microorganismos gram-positivos en Argentina fueron comparables a los registrados en países desarrollados, los de los bacilos gram-negativos frente a antibióticos como los $\beta$-lactámicos, aminoglucósidos y trimetoprima-sulfametoxazol triplicaron los observados en Europa y Estados Unidos.

La emergencia de resistencia puede ser el resultado inevitable del uso de los antimicrobianos, pero hay pruebas de que en algunas regiones ocurre con mayor velocidad que en otras. La proporción de aislados resistentes es mayor en países en desarrollo, lo cual sugiere que las diferencias en las condiciones sanitarias y los patrones de uso de estos fármacos tienen un papel importante $(3,13)$.

Los microorganismos multirresistentes se seleccionan con mayor facilidad en unidades hospitalarias, particularmente en las salas de cuidados intensivos, en las que se emplean antibióticos de amplio espectro como terapia empírica inicial; por selecciones sucesivas emergen microorganismos con escasas alternativas terapéuticas.

La aplicación de programas de vigilancia como el descrito permite establecer estrategias locales y regionales para el uso de los antimicrobianos y centrar los esfuerzos para controlar los niveles de resistencia y detectar rápidamente nuevos mecanismos o el aumento significativo de los ya existentes (14).

Agradecimientos. Agradecemos a John Stelling, miembro de la División de Vigilancia y Control de Enfermedades Emergentes y de otras Enfermedades Transmisibles de la Organización Mundial de la Salud, la permanente actualización del programa computadorizado utilizado en el programa WHONET y a Thomas O'Brien, miembro del Centro Colaborador para la Vigilancia de la Resistencia a Antimicrobianos de la OMS, su continuo apoyo técnico al funcionamiento de este programa. Queremos agradecer, además, a. Ezequiel Tuduri Franco (INEI) su valiosa participación en el tratamiento informático de los datos.

\section{REFERENCIAS}

1. Stelling J, O'Brien T. Surveillance of antimicrobial resistance: The WHONET Program. Clin Infect Dis 1997 19;24(Supl. 1):157-168.

2. O'Brien T, Stelling J. WHONET: Removing obstacles to the full use of information about antimicrobial resistance. Diagn Microbiol Infect Dis 1996;25:163-168.

3. Murray B. Can antibiotic resistance be controlled? N Engl J Med 1994;330:1229-1230.

4. National Committee for Clinical Laboratory Standards. Performance Standards for Antimicrobial Disk Susceptibility Tests: Approved Standard. $6^{a}$ ed. National Committee for Clinical Laboratory Standards document M2-A6. Villanova, Pennsylvania: National Committee for Clinical Laboratory Standards; 1997.

5. National Committee for Clinical Laboratory Standards. Performance Standards for Antimicrobial Disk Susceptibility Tests: Approved Standard. $5^{\text {a }}$ ed. National Committee for Clinical Laboratory Standards document M2-A5. Villanova, Pennsylvania: National Committee for Clinical Laboratory Standards;1996.

6. Livermore D. $\beta$-lactamases in laboratory and clinical resistance. Clin Microbiol Rev 1995;8: 557-584.
7. Livermore D, Williams D. $\beta$-lactams: mode of action and mechanisms of bacterial resistance. En: Lorian V, ed. Antibiotics in laboratory medicine. $4^{\mathrm{a}}$ ed. Baltimore: Williams and Wilkins; 1996. pp. 502-578.

8. Rossi A, Lopardo H, Woloj M, Picandet AM, Mariño M, Galas M, et al. Non-typhoid Salmonella spp. resistant to cefotaxime. J Antimicrob Chemother 1995:36:697-702.

9. Rossi A, Galas M, Binztein N, Rivas M, Caffer MI, Corso A, et al. Unusual multiresistant Vibrio cholerae O1 El Tor in Argentina. Lancet 1993;342:1172-1173.

10. Bauernfeind A, Stemplinger I, Jungwirth $R$, Mangold P, Amann S, Akalin E, et al. Characterization of $\beta$-lactamase gene bla $a_{P E R-2}$ which encodes an extended-spectrum class A $\beta$ lactamase. Antimicrob Agents Chemother 1996;40:616-620.

11. Nordmann P, Naas T. Sequence analysis of PER-1 extended-spectrum $\beta$-lactamase from Pseudomonas aeruginosa and comparison with class A $\beta$-lactamases. Antimicrob Agents Chemother 1994;38:104-114.

12. Bauernfeind A, Stemplinger I, Jungwirth R, Ernst S, Casellas JM. Sequences of $\beta$-lactamase genes encoding CTX-M-1 (MEN-1) and CTXM-2 and relationship of their amino acid sequences with those of other $\beta$-lactamases. Antimicrob Agents Chemother 1996;40:509-513.

13. Weis SE, Slocum PC, Blais FX, King B, Nunn M, Matney GB, et al. The effect of directly observed therapy on the rates of drug resistance and relapse in tuberculosis. N Engl J Med 1994;330:1179-1184.

14. O'Brien TF. The global epidemic nature of antimicrobial resistance and the need to monitor and manage it locally. Clin Infect Dis 1997;24(Supl 1):2-8.

Manuscrito recibido el 5 de agosto de 1998 y aceptado para publicación, tras revisión, el 2 de junio de 1999. 
ABSTRACT The World Health Organization has implemented a surveillance program for antimicrobial resistance that is known as WHONET. In Argentina the program was developed through a network of 23 public and private hospitals that participate in national

Monitoring antimicrobial resistance in Argentina: The WHONET program, 1995-1996 and international quality-control programs. Between January 1995 and December 1996, the antimicrobial susceptibility of 16073 consecutive clinical isolates was determined, using the recommended standards of the National Committee for Clinical Laboratory Standards of the United States of America. More than half of the Escherichia coli urinary isolates were resistant to ampicillin and more than $30 \%$ to trimethoprim/ sulfamethoxazole (SXT). When the percentage of resistant isolates from outpatients (OPs) was compared to that observed in hospitalized patients (HPs), a marked difference in antimicrobial activity was noted in the case of gentamicin ( $2 \%$ from OPs resistant vs. $8 \%$ from HPs resistant), norfloxacin (2\% vs. 6\%), and third-generation cephalosporins (7\% vs. 15\%). Of the Klebsiella pneumoniae isolates recovered from blood cultures, $71 \%$ and $60 \%$ showed resistance to third-generation cephalosporins and to gentamicin, respectively. The overall rate of oxacillin resistance in Staphylococcus aureus was 39\%. Around half of the Enterococcus spp. isolates showed high resistance to aminoglycosides, but resistance to glycopeptides was not found. In Argentina, ampicillin and SXT were not suitable for treating diarrhea. Shigella flexneri had a higher number of isolates resistant to both of those drugs ( $87 \%$ and $74 \%$, respectively) than Sh. sonnei did (47\% and $71 \%$, respectively). About $40 \%$ of the Salmonella spp. isolated in pediatric hospitals were resistant to third-generation cephalosporins. When microorganisms causing bacterial meningitis were examined, Streptococcus pneumoniae showed a resistance rate of $18 \%$ to penicillin and Haemophilus influenzae a resistance rate of $19 \%$ to ampicillin. These rates are within the intermediate range reported for other countries of the Americas and for Europe. 